\title{
The Analysis and Implementation of Edge Detection Algorithms in Image Processing Based on Matlab
}

\author{
Yang Bao-liang ${ }^{1, a^{*}}$, Jin Feng-ming ${ }^{1, b}$, Lei Ming-dong ${ }^{1, c}$ \\ 1Chongqing University of Arts and Sciences Chongqing 402160 P. R. China \\ a* Email: 327132567@qq.com \\ b Email: 824418985@qq.com \\ c Email: 405351991@qq.com
}

Keywords: Image processing; Edge Detection; method of differential operator

Abstract: Based on machine vision image feature extraction, the main content extraction edge detection chamber features, based on the analysis of the basic theory and methods of edge detection, edge detection algorithm for several commonly used Sobel, Log and Canny, on which the algorithm is simulated by use of MA TLAB, analyzes the performance characteristics on their respective, given the various applications edge algorithm, the foundation for future engineering practice under the. Realization

\section{Introduction}

The Feature extraction of digital image is one of the main contents of the study of image processing, the factor of point, the line and surface as the main features of the image are the hot and difficult domain of research. The edge of line image appears in irregular structure and unstable phenomenon that is mutation point of the signal, which gives the position of image profile. The profile is often some important characteristics that we need in the image edge detection, which requires us to a image edge detection and extract it. The basic idea of edge detection is to detect the edge points in the image, and accordingly connect the edge points into a profile, which then divided the region ${ }^{[1-3]}$.

The main use of various edge detection algorithm to find the possible enhanced image edge pixel for extraction can greatly reduce the amount of information to be processed but retained the image object shape information, of which the commonly used in image edge detection operators are: Robert operator, Sobels operator, Prewitt operator, Log operator, Canny operator, SUSAN operator, and then the edge detection method based on morphology. Here through research and programming with Matlab simulation analysis of the above algorithm, we get the characteristics of various algorithms and the scope of application, and provide a reference for the right image edge detection technology. Between the adjacent regions of different gray value there is always the edge, which is the result of discrete gray value ${ }^{[4-5]}$. This discontinuity is usually easily detected by the method of derivative .Commonly used for edge detection are first-order derivative and two order derivative the commonly used Detection operators are differential operator, LOG operator and Canny operator ${ }^{[6]}$.

\section{Gradient edge operator}

Test operator is used when the jump of gray value property happens in the edges of the image, and the gradient reflects the strength of the edge while Gradient direction reflects the change of intensity of edge information. In image processing, a first order derivative can be achieved by gradient, gradient detection, also known as a derivative method.

Any point in image processing gradient is represented by a vector. The magnitude and the direction of this vector respectively:

$$
|\nabla F|=\sqrt{\frac{\partial f^{2}}{\partial x}+\frac{\partial f^{2}}{\partial x}}=\sqrt{F_{x}^{2}+F_{y}^{2}} \quad \theta=\arctan \left(\frac{F_{x}}{F_{y}}\right)
$$


The gradient amplitude represents the edge change intensity; gradient direction means the edge direction is vertical. In the actual process of image processing, we use the finite difference instead of a derivative or gradient.

(1) Roberts operator

In the process of image processing, we use a pair of mutually vertical difference to approximate the differential, Roberts operator use the pixel diagonally adjacent to define gradient, the horizontal and vertical gradient template below.

$$
W_{x}=\left(\begin{array}{ccc}
-1 & 0 & 0 \\
0 & 1 & 0 \\
0 & 0 & 0
\end{array}\right), W_{y}=\left(\begin{array}{ccc}
0 & -1 & 0 \\
1 & 0 & 0 \\
0 & 0 & 0
\end{array}\right),
$$

In ATLAB using Roberts edge detection operator, in the format: [J, thresh]=edge(I, 'roberts', $35 / 255$ ) and the effect is shown in the following figure.

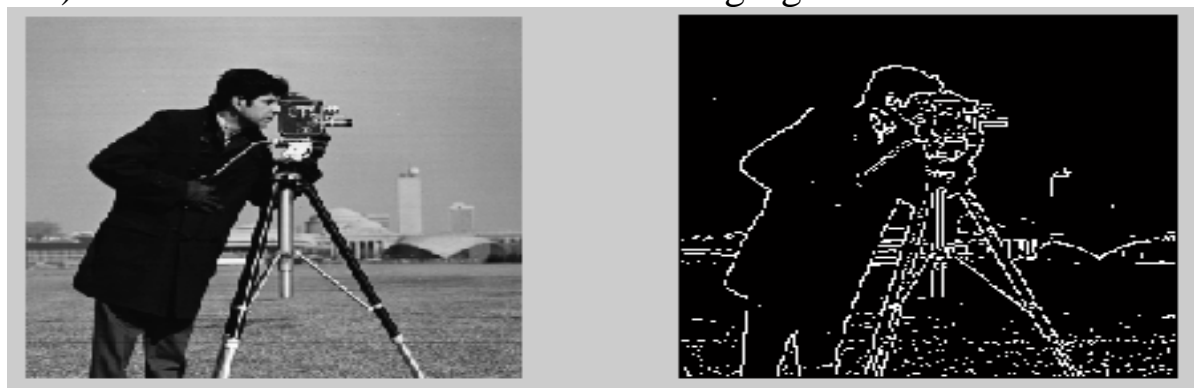

Figure1 the image of Roberts operator edge detection

As shown by the picture, Roberts makes use of the gradient operation of four pixel equivalent, which is simple but the edge detection is not continuous, and is sensitive to the noise, thus the algorithm is applied to the image edge without noise processing.

(2) Prewitt operator

Due to the Sensitivity to noise of Roberts operator using four point difference method, we use the 8 point difference approximation of the gradient method to reduce the noise interference, we generally use the average difference gradient, the horizontal and vertical gradient template below.

$$
W_{x}=\frac{1}{3}\left(\begin{array}{lll}
-1 & 0 & 1 \\
-1 & 0 & 1 \\
-1 & 0 & 1
\end{array}\right), W_{y}=\frac{1}{3}\left(\begin{array}{ccc}
-1 & -1 & -1 \\
0 & 0 & 0 \\
1 & 1 & 1
\end{array}\right),
$$

In ATLAB using Prewitt edge detection operator, in the format[J, thresh]=edge(I, 'prewitt', [], 'both') and the effect is shown in the following figure.

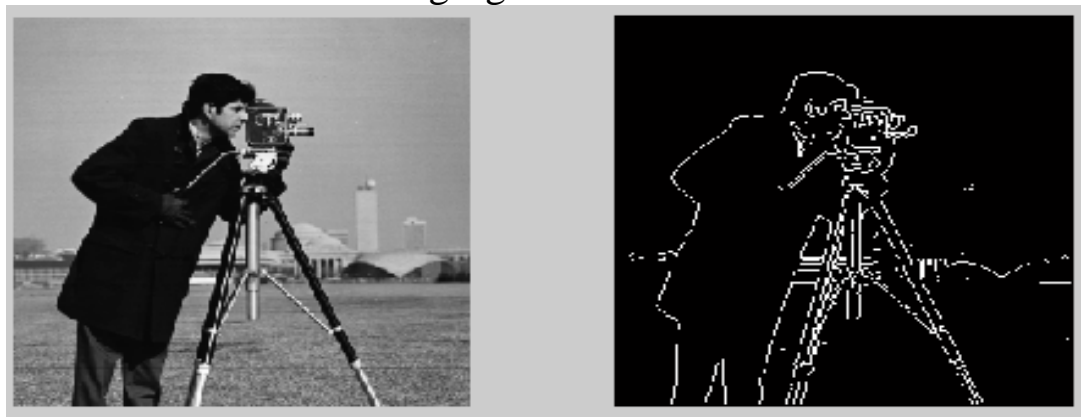

Figure 2 the image of prewitt operator edge detection

As shown by the Prewitt operator, the gradient operation of 8 pixel equivalent, at the same time average of pixels, from the experimental results, the noise is more obviously ristricted, but some of the edge information is not correctly recognized.

(3) Sobel operator

Prewitt operator equally divide the pixels, resulting in some edge which can not be identified, so we zoom in some pixels and and then equally divide the pixels, which formed the Sobel operator. In order to achieve good effect, we only deal with the pixel recently performed on the amplification coefficient, we choose 2 , so the gradient template the horizontal and vertical directions respectively: 


$$
W_{x}=\frac{1}{4}\left(\begin{array}{lll}
-1 & 0 & 1 \\
-2 & 0 & 2 \\
-1 & 0 & 1
\end{array}\right), W_{y}=\frac{1}{4}\left(\begin{array}{ccc}
-1 & -2 & -1 \\
0 & 0 & 0 \\
1 & 2 & 1
\end{array}\right),
$$

In ATLAB using Sobel edge detection operator, in the format [J, thresh]=edge(I, 'sobel', [], 'both') and the effect is shown in the following figure.

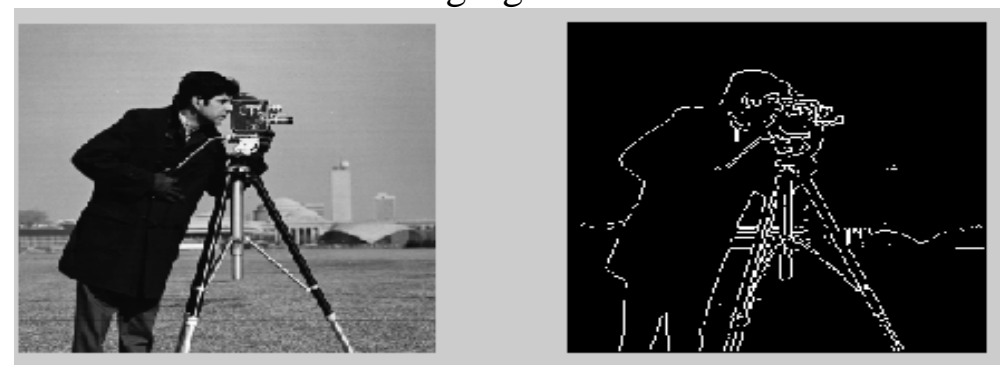

Figure3 the image of sobel operator edge detection

As shown by the Sobel operator, using a 8 pixel to do enlarging and dividing operation can effectively restrain the noise, edge extraction, thus the improved Sobel operator can better process image on the gray gradient and noise .

\section{The two derivative operator}

Two order differential operator makes use of the zero derivative of images appearing at the edge, that is to use different symbols in the two order derivative of the edge points to extract edge points.

(1) Laplacian operator

In digital image processing, Laplacian operator is defined at any point is:

$$
W=\left(\begin{array}{ccc}
0 & -1 & 0 \\
-1 & 4 & -1 \\
0 & -1 & 0
\end{array}\right)
$$

In ATLAB using Laplacian edge detection operator, in the format [J, thresh]=edge(I, 'zerocross', 0.007)and the effect is shown in the following figure.

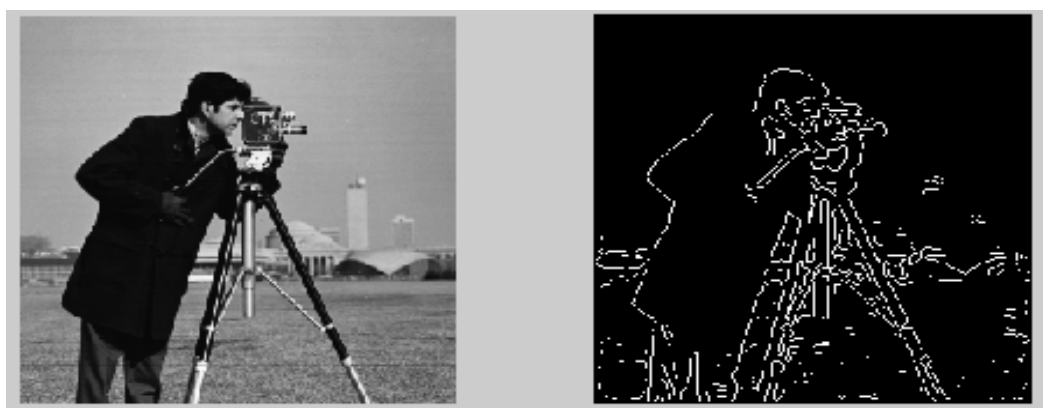

Figure4 the image of Laplacian operator edge detection

As shown by the above, image is obtained by using the Laplacian operator, is sensitive to noise, so in the digital image processing it is rarely used.

(2) LOG operator

The Laplacian operator in the above image is sensitive to noise, in order to solve the unsettled problem, we use the Gaussian to filter image and, and then use the Laplacian operator for edge detection , which can avoid the sensitivity of Laplacian to noise, implementation of the principle of this method is LOG operator. as we know The Gauss function in linear system, using Gauss function for image filter and two order differential operator is equivalent to the first of the two order differential Gauss function, then the image convolution operation, the expression of LOG operator is: 


$$
\nabla^{2} G(\mathrm{x}, \mathrm{y})=\frac{\partial^{2} G(\mathrm{x}, \mathrm{y})}{\partial x^{2}}+\frac{\partial^{2} G(\mathrm{x}, \mathrm{y})}{\partial y^{2}}=\left[\frac{x^{2}+y^{2}-2 \delta^{2}}{\delta^{4}}\right] \mathrm{e}^{-}\left(\frac{x^{2}+y^{2}}{2 \delta^{2}}\right)
$$

In the application of LOG operator, in Gauss's function the choice of the standard deviation of parameters is very important, and is the key point for the operator in image processing. When the value of $\delta$ is small, image is smooth, and vise, image sharpening. The expression of the template is shown in the following formula.

$$
\left[\begin{array}{ccccc}
0 & 0 & -1 & 0 & 0 \\
0 & -1 & -2 & -1 & 0 \\
-1 & -2 & 16 & -2 & -1 \\
0 & -1 & -2 & -1 & 0 \\
0 & 0 & -1 & 0 & 0
\end{array}\right]
$$

In ATLAB using LOG edge detection operator, in the format[K, thresh]=edge(J, 'log', [], 2.3) and the effect is shown in the following figure.

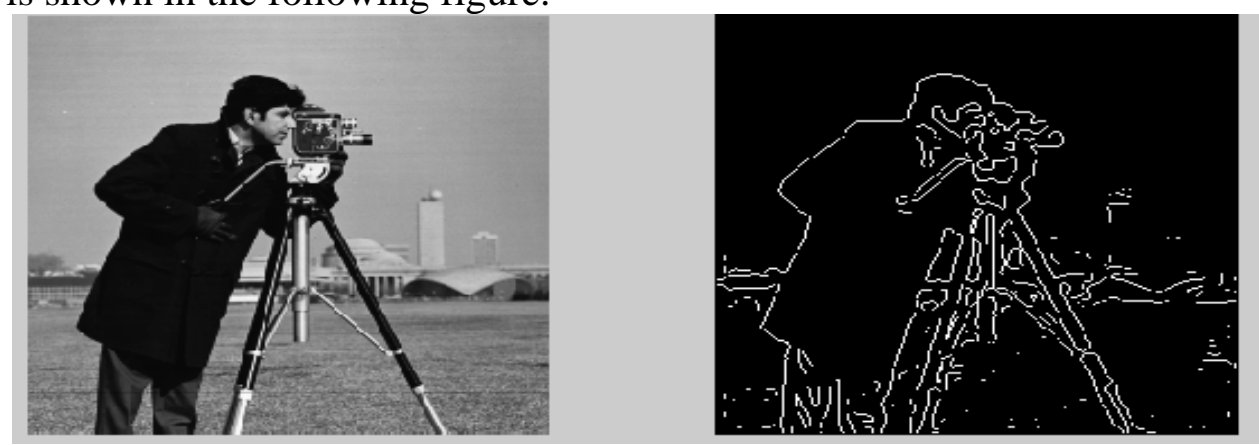

Figure5 the image of LOG operator edge detection

\section{Summary}

From the simulation results can be seen intuitively, each algorithm has its advantages. Therefore, in practical application, satisfactory results can be achieved by analysis of concrete problems and the choice of the best edge detection operator according to the specific circumstances. The classical differential operator theory and the template is simple and easy to operate to detect more accurate positioning and a clear edge, but the details are not complete while the number of edge detection in graph by Robert, Sobel and prewitt operator are incomplete, and it is sensitive to noise. The LOG operator has good continuity with a dominant integrity. In contrast, the LOG operator's edge is thicker, but with more noise points, with best edge linking to show the most clear details and very comprehensive outline of the edge extraction. Based on the detailed analysis of the several typical edge detection operator, it provide scientific theoretical basis for future research and practical application, which has a certain reference value.

\section{Acknowledgements}

This work was supported by the Chongqing University of Arts and Sciences Foundation (Grant No: Z2014DQ06), and Chongqing University of Arts and Sciences Foundation of Student(Grant No: XSKY2015049).

\section{References}

[1] Wei Wei. Comparative Study on Some Common Edge Detection Methods Based on Matlab [J]. Modern Electronics Technique.2011,34(4):91-94. 
[2] Li Tingjiao, Cai Zhenjiang, Wang Wendi. Edge Extraction of Cow's Digital Image Based on MATLAB[J]. Journal of Agricultural Mechanization Research.2011， 2(2):23-26.

[3] YANG Xian-Hua. Study on Edge Detection Algorithm in Image Processing[J]. Development Innovation of Machinery Electrical Products. 2010, 23(2):129-131.

[4] WANG Xiao-jun, LIU Xu-min. Image Edge Detection Algorithm Based on Improved Canny Operator[J]. Computer Engineering.2012,38(14):196-202.

[5] LI Ya-ya, LI Zhi-jie. Comparison and implementation of image edge detection algorithm[J]. Computer Engineering and Design.2010,31(9):1971-1975.

[6] LU Xingjuan ,WU Zhenyu. Research on Technique of Image Edge Detection[J]. Modern Electronics Technique.2010,317(6):128-130. 